\title{
DanceDanceThumb: Tablet App for Rehabilitation for Carpal Tunnel Syndrome
}

\author{
Takuro Watanabe ${ }^{1 \dagger}$, Yuta Sugiura ${ }^{1}$, Natsuki Miyata ${ }^{2}$, \\ Koji Fujita $^{3}$, Akimoto Nimura ${ }^{3}$, and Maki Sugimoto ${ }^{1}$ \\ ${ }^{1}$ Keio University, 3-14-1 Hiyoshi, Kohoku, Yokohama, Kanagawa, 223-8522, Japan \\ \{t.tawatana, sugiura, sugimoto\}@imlab.ics.keio.ac.jp \\ ${ }^{2}$ National Institute of Advanced Industrial Science and Technology, 2-3-26 Aomi, Koto, \\ Tokyo, 135-0064, Japan \\ n.miyata@aist.go.jp \\ ${ }^{3}$ Tokyo Medical and Dental University, 1-5-45 Yushima, Bunkyo, Tokyo, 113-8510, Japan \\ \{fujiorth, nimura.orj\}@tmd.ac.jp
}

\begin{abstract}
We propose a tablet app that aids in rehabilitation for carpal tunnel syndrome. Patients play a game on a tablet PC in which they catch animal characters displayed on the screen with their thumb. In addition, we developed a system that records patient rehabilitation logs from the tablet and sends them to the cloud, where doctors can observe a patient's condition remotely.
\end{abstract}

Keywords: Rehabilitation · Carpal Tunnel Syndrome · Gamification.

\section{Introduction}

Thumbs make it possible to grasp and pick stuff up, such as pens, with other fingers are very important. However, the aging process and applying too much daily stress on the fingers lead to convulsions in the carpal tunnel, causing paralysis of the thumbs that makes it difficult to move them. This condition is called "carpal tunnel syndrome" (CTS).

For CTS patients, doctors recommend using the thumbs daily for rehabilitation. However, because rehabilitation programs are often boring, it is difficult for patients to remain motivated. As a result, problems occur, such as the thumbs having a limited range of motion because there has not been enough exercise.

In this paper, we propose a tablet application that supports CTS rehabilitation. CTS patients play a game in which they catch animal characters displayed on the screen with their thumb. We also develop a system that records patient rehabilitation logs on a cloud system, so doctors can observe a patient's condition remotely. 


\section{Related Work}

Ploderer et al. developed a system that visualizes body movement during rehabilitation and analyzes performance by using a heat map display used with wearable sensors attached to multiple parts of the human body [1]. While the system can accurately measure a user's performance, there is the possibility that troublesome preparation will be required to use it, and body movement could be impeded. Kadomura et al. proposed a system that improves the motivation to exercise by measuring body movement with Microsoft Kinect and sharing it with people in remote places [2]. Kinect does not impede body movement or require attaching sensors to the body, which can be troublesome. However, there is the possibility that objects such as furniture may become an occlusion between Kinect and users. Also, these applications target whole body exercise, there is no rehabilitation specialized in finger movement.

There is a smartphone app that collects patient's use logs and analyzes neurological disorders [3]. However, this is specialized for estimating diseases and does not aim to aid in rehabilitating patients suffering from them.

\section{DanceDanceThumb}

\subsection{Overview of System}

In this paper, we propose DanceDanceThumb, a tablet app that supports the rehabilitation process for CTS patients. We developed a game in which players collect animal characters by using a simple user interface because most patients with CTS are elderly. We aim for patients to maintain the rehabilitation process with this app. In addition, the app can measure the range of motion of the thumb. Patients can see how much of the ability of their thumb has been recovered by looking at the range of motion of the thumb recorded on the tablet.

\subsection{Flow of Experience}

First, the patient inserts and fixes their other fingers into a finger guide. Next, the patient measures the extent of thumb movement by drawing a "circle" with the thumb. The circle is recorded in the cloud as two-dimensional coordinates. In addition, the app uses this circle data to set the difficulty of the game. The patient touches any point on the screen with the thumb.

When the patient touches the screen with the thumb, a small green circle is displayed around the position of the thumb (Fig. 1, left). The size of this green circle is approximated to the registered circle at the beginning. At the same time, an illustration of the thumb appears in a large green circle. Thumb movements made in the small green circle are amplified and displayed on the large circle. This makes it easier for patients to understand their own movement because the range of thumb motion after surgery is extremely small. The movement of the thumb is expanded three times and reflected in the illustration of the thumb in the large circle. By controlling the 
illustration, the patient collects animal characters that appear until they reach a set number. When the patient plays again, the range of thumb motion is re-measured.

\subsection{Implementation}

We created a finger guide that is attached to the upper part of a tablet in order to fix fingers except the thumb (Fig.1 left). The guide was designed so that the distance between each finger was $2 \mathrm{~cm}$, and it is printed by using a 3D printer.

We developed the app with Unity software (Fig.1 right). The touch position of thumb on the touch screen is recorded to a database as 2-dimensional coordinates. The app displays the results of daily rehabilitation with a monthly calendar. The calendar shows the total rehabilitation time for each day with numbers and color gradations. The database was developed by using an existing cloud service, NIFTY Cloud. Recorded data are stored in this database.

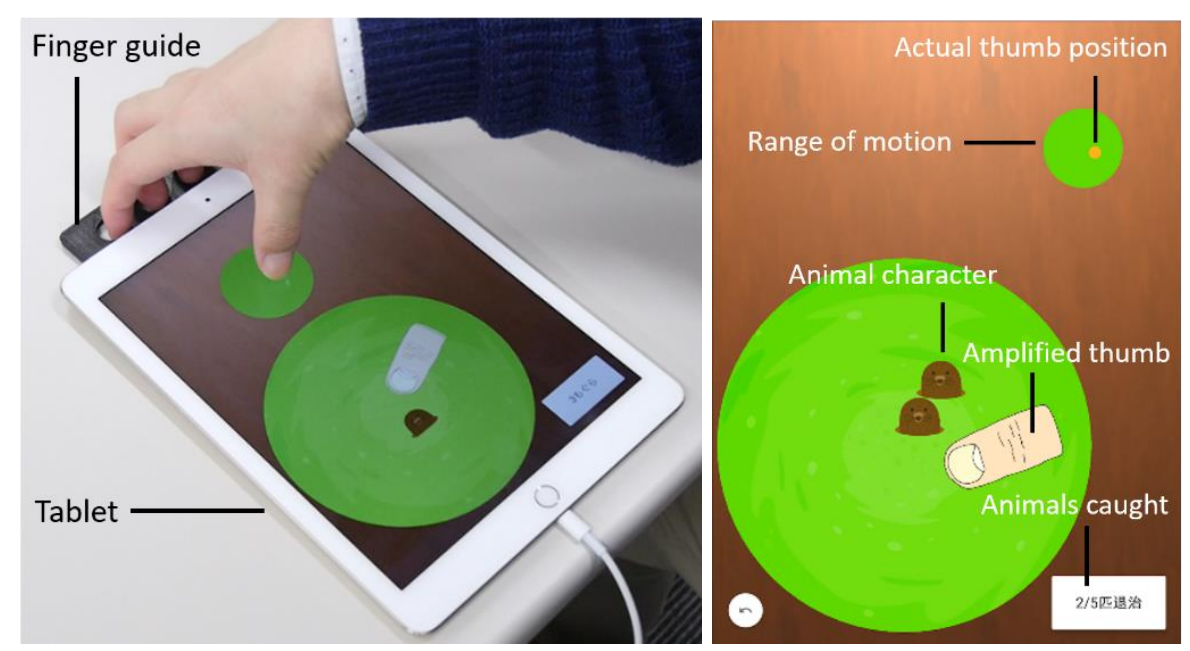

Fig. 1. Game screen of DanceDanceThumb

\section{$4 \quad$ User Trial}

We are currently conducting a user trial with actual patients with CTS. All subjects provided full informed consent, and the study was approved by the Tokyo Medical and Dental University Review Board. They have been able to use the system well so far. Hereafter, we are going to collect data from them for 2 months. Figure 2 shows a patient using the app. 


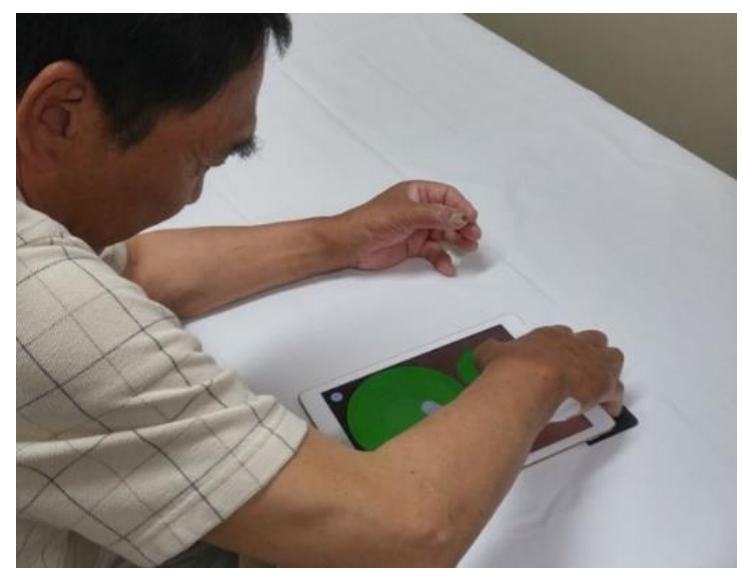

Fig. 2. Actual patient using app

\section{$5 \quad$ Conclusion and Future Work}

We developed a tablet app that aids in self-rehabilitation for CTS patients. By exercising the thumb on the screen to play a game, patients can become rehabilitated by themselves. At this time, we focused on CTS related conditions and developed the app for CTS patients.

As future work, we will observe how much our app contributes to keeping patients motivated to continue with the rehabilitation process. Moreover, we will examine whether the app is significant for recovering movement performance. We also plan to develop apps to support rehabilitation for other orthopedic diseases.

\section{Acknowledgements}

This work was supported by JSPS KAKENHI Grant Numbers JP26700017 and JP16H01741.

\section{References}

1. Azusa Kadomura, Akira Matsuda, and Jun Rekimoto. 2016. CASPER: A Haptic Enhanced Telepresence Exercise System for Elderly People. In Proc. AH '16. ACM, Article 2, 8 pages.CONFERENCE 2016, LNCS, vol. 9999, pp. 1-13. Springer, Heidelberg (2016).

2. Bernd Ploderer, Justin Fong, Anusha Withana, Marlena Klaic, Siddharth Nair, Vincent Crocher, Frank Vetere, and Suranga Nanayakkara. 2016. ArmSleeve: A Patient Monitoring System to Support Occupational Therapists in Stroke Rehabilitation. In Proc. DIS '16. ACM, 700-711.

3. MONTFORT YOUR PERSONAL CHECKUP, http://www.mon4t.com/ 